\title{
Chronic Cutaneous Graft Versus Host Disease
}

National Cancer Institute

\section{Source}

National Cancer Institute. Chronic Cutaneous Graft Versus Host Disease. NCI Thesaurus.

Code C160874.

Chronic graft versus host disease (GvHD) occurring in the skin. 